\title{
Correction to: Proton pump inhibitor monotherapy is effective to attenuate dyspepsia symptoms associated with gastroesophageal reflux disease: a multicenter prospective observational study
}

\author{
Kimio Isshi ${ }^{1,2}$ - Nobuyuki Matsuhashi ${ }^{3}$ Takashi $\mathrm{Joh}^{4} \cdot$ Kazuhide Higuchi $^{5}$. \\ Katsuhiko Iwakiri $^{6}$ - Takeshi Kamiya ${ }^{7}$ - Noriaki Manabe ${ }^{8}$ Maiko Ogawa9 \\ Seiji Arihiro ${ }^{9} \cdot$ Ken Haruma ${ }^{10} \cdot$ Koji Nakada $^{11}$
}

Published online: 11 March 2019

(C) Japanese Society of Gastroenterology 2019

\section{Correction to: J Gastroenterol \\ https://doi.org/10.1007/s00535-019-01546-0}

In the original publication of this article, Tables 2, 3, 4, 5 and 6 were published incorrectly. The correct tables are given in this correction.

The original article can be found online at https://doi.org/10.1007/ s00535-019-01546-0.

Kimio Isshi

isshi-ki@jikei.ac.jp

1 Isshi Gastro-Intestinal Clinic, 2-15-21, Shinozaki-Cho, Edogawa-ku, Tokyo 133-0061, Japan

2 Department of Endoscopy, Jikei University School of Medicine, 3-15-8 Nishishinbashi, Minato-Ku,

Tokyo 105-8461, Japan

3 Department of Gastroenterology, NTT Medical Center Tokyo, 5-9-22, Higashi-Gotanda, Shinagawa-Ku, Tokyo 105-8461, Japan

4 Gamagori City Hospital, 1-1 Mukaida, Hirata-Cho, Gamagori-City, Aichi 443-8501, Japan

5 Second Department of Internal Medicine, Osaka Medical College, 2-7 Daigaku-machi, Takatsuki, Osaka 569-8686, Japan

6 Department of Gastroenterology, Nippon Medical School Graduate School of Medicine, 1-1-5, Sendagi, Bunkyo-Ku, Tokyo 133-8603, Japan
7 Department of Medical Innovation, Nagoya City University Graduate School Medical Sciences,

1, Kawasumi, Mizuho-Cho, Mizuho-Ku, Nagoya 467-8601, Japan

8 Division of Endoscopy and Ultrasonography, Department of Laboratory Medicine, Kawasaki Medical School General Medical Center, 2-6-1, Nakayamashita, Kita-Ku, Okayama-City, Okayama 700-8505, Japan

9 Division of Gastroenterology and Hepatology, Department of Internal Medicine, Katsushika Medical Center Jikei University School of Medicine, 6-41-2, Aoto, Katsushika-Ku, Tokyo 125-8506, Japan

10 Department of General Internal Medicine 2, Kawasaki Medical School Kawasaki Hospital, 577, Matsushima, Kurashiki, Okayama 701-0192, Japan

11 Department of Laboratory Medicine, The Jikei University Daisan Hospital, 4-11-1, Izumihon-Cho, Komae-City, Tokyo 201-8601, Japan 
Table 2 Patients' characteristics

\begin{tabular}{|c|c|c|c|c|c|c|c|c|}
\hline & $\begin{array}{l}\text { Total } \\
(n=233)\end{array}$ & $\begin{array}{l}\text { GERD-SS } \leqq 2.5 \\
\text { mild symptom } \\
(n=40)\end{array}$ & $\begin{array}{l}\text { GERD-SS } 3.0-4.5 \\
\text { moderate symptom } \\
(n=151)\end{array}$ & $\begin{array}{l}\text { GERD-SS } \geqq 5.0 \\
\text { severe symptom } \\
(n=42)\end{array}$ & $\begin{array}{l}p- \\
\text { value }\end{array}$ & $\begin{array}{l}\text { Mild vs } \\
\text { moderate } \\
p \text {-value }\end{array}$ & $\begin{array}{l}\text { Moderate } \\
\text { vs severe } \\
p \text {-value }\end{array}$ & $\begin{array}{l}\text { Mild vs } \\
\text { severe } \\
p \text {-value }\end{array}$ \\
\hline Age (year) ${ }^{*}$ & $56.5 \pm 14.1$ & $56.7 \pm 15.5$ & $56.9 \pm 13.0$ & $54.7 \pm 16.4$ & $0.675^{\mathrm{a}}$ & 0.996 & 0.652 & 0.804 \\
\hline $\operatorname{BMI}\left(\mathrm{kg} / \mathrm{m}^{2}\right)^{*}$ & $23.7 \pm 3.7$ & $22.3 \pm 3.3$ & $23.8 \pm 3.5$ & $24.7 \pm 4.5$ & $0.014^{\mathrm{a}}$ & 0.054 & 0.395 & 0.012 \\
\hline Gender: $n(\%)$ & & & & & $0.007^{\mathrm{b}}$ & & & \\
\hline Male & $136(58)$ & $29(73)$ & $87(58)$ & $20(48)$ & & & & \\
\hline Female & $97(42)$ & $11(28)$ & $64(42)$ & $22(52)$ & & & & \\
\hline \multicolumn{9}{|c|}{ Endoscopic finding: $n(\%)$} \\
\hline NERD/ERD & & & & & $0.725^{\mathrm{b}}$ & & & \\
\hline NERD & $94(41)$ & $18(45)$ & $58(39)$ & $18(43)$ & & & & \\
\hline ERD & $138(59)$ & $22(55)$ & $92(61)$ & $24(57)$ & & & & \\
\hline LA classification & & & & & $0.916^{\mathrm{b}}$ & & & \\
\hline Grade N & $58(25)$ & $12(30)$ & $34(23)$ & $12(29)$ & & & & \\
\hline Grade M & $36(16)$ & $6(15)$ & $24(16)$ & $6(14)$ & & & & \\
\hline Grade A & $71(31)$ & $12(30)$ & $45(30)$ & $14(33)$ & & & & \\
\hline Grade B & $49(21)$ & $7(18)$ & $36(24)$ & $6(14)$ & & & & \\
\hline Grade C & $16(7)$ & $3(8)$ & $9(6)$ & $4(10)$ & & & & \\
\hline Grade D & $2(1)$ & $0(0)$ & $2(1)$ & $0(0)$ & & & & \\
\hline
\end{tabular}

GERD gastroesophageal reflux disease, $S S$ symptom subscale, $B M I$ body mass index, NERD non-erosive reflux disease, ERD erosive reflux disease, LA Los Angeles

*Data are presented as mean $\pm \mathrm{SD}$

a analysis of variance (ANOVA)

${ }^{\mathrm{b}}$ Chi-square test

${ }^{\mathrm{c}}$ Tukey test

Table 3 Comparison of the GERD and FD symptom scores at baseline among the groups divided by the severity of GERD-SS before PPI therapy

\begin{tabular}{|c|c|c|c|c|c|c|c|c|c|c|c|}
\hline & \multirow{2}{*}{$\begin{array}{l}\text { Total } \\
(n=233)\end{array}$} & \multirow{2}{*}{$\begin{array}{l}\text { GERD-SS } \\
\leqq 2.5 \\
\text { mild } \\
\text { symptom } \\
(n=40)\end{array}$} & \multirow{2}{*}{$\begin{array}{l}\text { GERD-SS } \\
3.0-4.5 \\
\text { moderate } \\
\text { symptom } \\
(n=151)\end{array}$} & \multirow{2}{*}{$\begin{array}{l}\text { GERD-SS } \\
\geqq 5.0 \\
\text { severe } \\
\text { symptom } \\
(n=42)\end{array}$} & \multirow[t]{2}{*}{$p$-value ${ }^{\mathrm{a}}$} & \multicolumn{2}{|c|}{ Mild vs moderate } & \multicolumn{2}{|c|}{ Moderate vs severe } & \multicolumn{2}{|c|}{ Mild vs severe } \\
\hline & & & & & & $p$-value ${ }^{\mathrm{b}}$ & $\begin{array}{l}\text { Cohen's } \\
d^{c}\end{array}$ & $p$-value ${ }^{\mathrm{b}}$ & $\begin{array}{l}\text { Cohen's } \\
d^{\mathrm{c}}\end{array}$ & $p$-value ${ }^{\mathrm{b}}$ & $\begin{array}{l}\text { Cohen's } \\
d^{\text {c }}\end{array}$ \\
\hline GERD-SS* & $3.7 \pm 1.1$ & $2.1 \pm 0.4$ & $3.6 \pm 0.5$ & $5.5 \pm 0.6$ & $<0.001$ & $<0.001$ & 2.88 & $<0.001^{\mathrm{b}}$ & 3.42 & $<0.001^{\mathrm{b}}$ & 6.54 \\
\hline FD-SS* & $3.3 \pm 0.9$ & $2.6 \pm 0.6$ & $3.2 \pm 0.7$ & $4.3 \pm 1.1$ & $<0.001$ & $<0.001$ & 0.79 & $<0.001^{\mathrm{b}}$ & 1.43 & $<0.001^{\mathrm{b}}$ & 1.99 \\
\hline FD-EPS-Sx* & $3.6 \pm 1.2$ & $2.6 \pm 1.2$ & $3.5 \pm 0.9$ & $4.8 \pm 1.3$ & $<0.001$ & $<0.001$ & 0.91 & $<0.001^{\mathrm{b}}$ & 1.28 & $<0.001^{\mathrm{b}}$ & 1.81 \\
\hline FD-PDS-SS* & $3.0 \pm 1.2$ & $2.7 \pm 1.0$ & $2.9 \pm 1.0$ & $3.9 \pm 1.3$ & $<0.001$ & 0.521 & 0.21 & $<0.001^{\mathrm{b}}$ & 0.91 & $<0.001^{\mathrm{b}}$ & 1.05 \\
\hline
\end{tabular}

SS symptom subscale, EPS epigastric pain syndrome, $S x$ symptom, $P D S$ postprandial distress syndrome

*Data are presented as mean $\pm \mathrm{SD}$

${ }^{a}$ Analysis of variance (ANOVA)

${ }^{\mathrm{b}}$ Tukey test

'Interpretation of effect size (Cohen's $d$ ); $\geqq 0.2$ :small, $\geqq 0.5$ :medium, $\geqq 0.8$ :large 

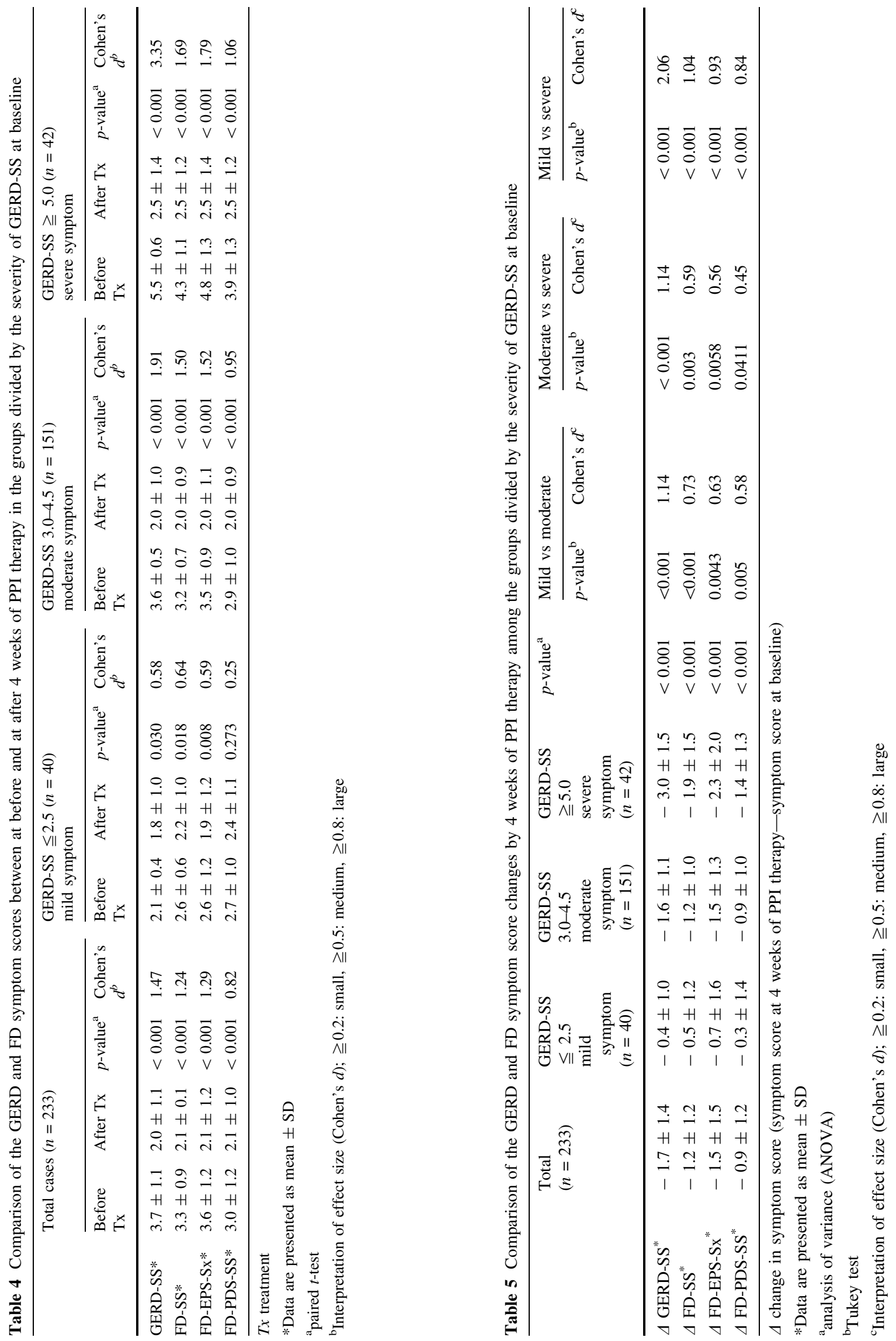
Table 6 Effect of factors on FD-SS score changes by 4 weeks of PPI therapy $(n=233)$

\begin{tabular}{lll}
\hline Multiple regression analysis & & \\
\hline Factor & $\beta^{*}$ & $p$-value \\
\hline Endoscopic findings (NERD) & 0.090 & 0.1355 \\
GERD-SS & -0.411 & $<0.001$ \\
$R^{2 * *}$ & 0.173 & $<0.001$ \\
\hline Interpretation of effect size & $\beta$ & $R^{2}$ \\
\hline None or very small & $<0.100$ & $<0.020$ \\
Small & $\geqq 0.100$ & $\geqq 0.020$ \\
Medium & $\geqq 0.300$ & $\geqq 0.130$ \\
Large & $\geqq 0.500$ & $\geqq 0.260$
\end{tabular}

$* \beta$ standardized regression coefficient

$* * R^{2}$ : coefficient of determination 